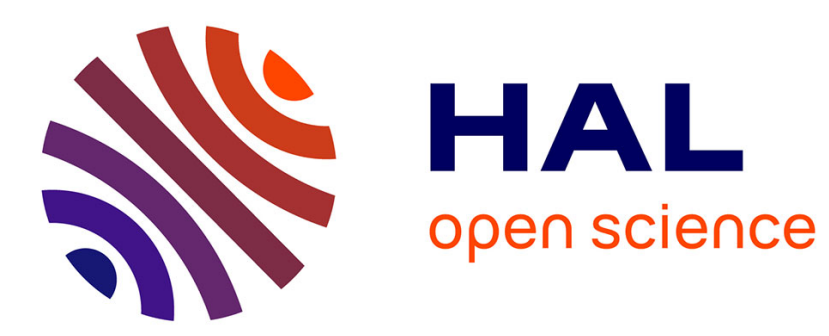

\title{
Manipulating the ferroelectric polarization state of BaTiO 3 thin films
}

Sukanya Datta, Maxime Rioult, Dana Stanescu, Hélène Magnan, Antoine Barbier

\section{- To cite this version:}

Sukanya Datta, Maxime Rioult, Dana Stanescu, Hélène Magnan, Antoine Barbier. Manipulating the ferroelectric polarization state of BaTiO 3 thin films. Thin Solid Films, 2016, 607, pp.7. 10.1016/j.tsf.2016.03.059 . cea-01369284

\section{HAL Id: cea-01369284 https://hal-cea.archives-ouvertes.fr/cea-01369284}

Submitted on 20 Sep 2016

HAL is a multi-disciplinary open access archive for the deposit and dissemination of scientific research documents, whether they are published or not. The documents may come from teaching and research institutions in France or abroad, or from public or private research centers.
L'archive ouverte pluridisciplinaire HAL, est destinée au dépôt et à la diffusion de documents scientifiques de niveau recherche, publiés ou non, émanant des établissements d'enseignement et de recherche français ou étrangers, des laboratoires publics ou privés. 


\title{
Manipulating the ferroelectric polarization state of $\mathrm{BaTiO}_{3}$ thin films
}

\author{
S. Datta, M. Rioult, D. Stanescu, H. Magnan, A. Barbier* \\ Service de Physique de l'Etat Condensé, SPEC, CEA, CNRS, Université Paris-Saclay, CEA Saclay, 91191 Gif-sur-Yvette Cedex, France
}

\section{A R T I C L E I N F O}

\section{Article history:}

Received 14 October 2015

Received in revised form 8 March 2016

Accepted 29 March 2016

Available online 31 March 2016

\section{Keywords:}

Ferroelectric thin films

Macroscopic polarization

Microscopic polarization

Atomic oxygen molecular beam epitaxial

method (AO-MBE)

Piezo response and atomic force microscopy

(PFM and AFM)

Annealing

Overcurrent

\begin{abstract}
A B S T R A C T
Controlling the ferroelectric polarization at macroscopic or microscopic levels is crucial in the framework of the development of ferroelectric materials used in yet challenging photo-electrochemical (PEC) cells and spintronic applications. We report here on polarization methods allowing to electrically polarize prototypical samples of $\mathrm{BaTiO}_{3}$ (001) films. Epitaxial single crystalline layers were grown up to a thickness of $25 \mathrm{~nm}$ by atomic oxygen assisted molecular beam epitaxy on 1 at.\% $\mathrm{Nb}_{\text {doped }} \mathrm{SrTiO}_{3}(001)$ single crystals. The samples were both microscopically and macroscopically polarized using Piezoresponse Force Microscopy and electrochemical poling in an electrolyte respectively. In addition we demonstrate the possibility to retrieve a quasi-native mixed ferroelectric polarization state after annealing. These polarization methods may be applied to many other ferroelectric thin films.
\end{abstract}

(C) 2016 Elsevier B.V. All rights reserved.

\section{Introduction}

There is currently a strong interest towards developing ferroelectric materials for various applications such as their use in multiferroics systems [1-4], photoelectrochemical cells (PEC) [5-8], and heat and gas sensors [9-11]. Ferroelectricity is a particular property of non-centrosymmetric materials exhibiting a spontaneous electrical polarization that can be reversed by the application of an external electric field. In the recent years, an impressive amount of contributions have focused on the ferroelectric properties of perovskite-based oxides such as $\mathrm{BiFeO}_{3}, \mathrm{BaTiO}_{3}$, PZT (Lead Zirconium Titanate) [12-17]. For such crystals, in the ferroelectric state, the barycenter of cations and anions in the unit cell are slightly spatially separated, inducing an electric dipole, i.e. an electric polarization. Dipoles rearrange themselves to minimize the sample energy forming different ferroelectric domains wherein the electric polarization points towards a given direction. Usually in an as-grown thin ferroelectric film the polarization is not uniform and the sample exhibits several ferroelectric domains where the polarization vector is pointing towards different directions. However, it is possible to control the orientation of the domains through the application of a saturating external electric field that allows the majority of the electrical dipoles within a domain to be oriented in a particular direction. Because of the wealth of technologically important applications deriving from the inclusion of ferroelectric layers in smart devices, it is of major

\footnotetext{
* Corresponding author.

E-mail address: antoine.barbier@cea.fr (A. Barbier).
}

importance to understand and master the manipulation of the domains electric polarization orientation in thin films, at the microscopic and macroscopic scales. Recently multiferroic $\mathrm{BiFeO}_{3}$ samples were considered within this framework. M. Singh et al. have reported on the local polarization of $\mathrm{BiFeO}_{3}$ samples at the microscopic scale by applying a $10 \mathrm{~V}$ potential on an AFM tip [18]. Cao et al. have independently demonstrated the poling of $\mathrm{BiFeO}_{3}$ samples at the macroscopic scale in a solution of Lithium Chlorate $\left(\mathrm{LiClO}_{4}\right)$ in propylene carbonate [19]. In the present work we consider the prototypical ferroelectric material $\mathrm{BaTiO}_{3}$ that has the major advantages as exhibiting a simple perovskite crystalline structure and featuring a para- to ferro-electric transition at relatively high temperature $\left(130{ }^{\circ} \mathrm{C}\right.$ for bulk $\mathrm{BaTiO}_{3}$ and even much higher for strained layers [20]). Here we demonstrate routes to master the manipulation of the polarization state at microscopic and macroscopic scales of $\mathrm{BaTiO}_{3} / \mathrm{Nb}: \mathrm{SrTiO}_{3}$ samples, as well as the possibility to erase an applied polarization state by an air annealing of the very same samples.

\section{Experimental details}

The $\mathrm{BaTiO}_{3}$ epitaxial layers were deposited on single crystalline 1 at.\% $\mathrm{Nb}: \mathrm{SrTiO}_{3}(001)$ (i.e. $~ 0.5 \mathrm{wt} \% \mathrm{Nb}$ ) substrates using atomic oxygen assisted molecular beam epitaxy (AO-MBE), a technique that makes possible the deposition of single crystalline layers of controlled morphology, stoichiometry and thickness [21]. The doped substrate is conductive enough to use it as electrode during the $\mathrm{BaTiO}_{3}$ overlayer poling procedures. The oxide layers were formed by thermal 
evaporating high purity metals from Knudsen cells, while exposing the sample to an atomic oxygen plasma (RF power $=350 \mathrm{~W}$ ) in an ultrahigh vacuum vessel $\left(10^{-7}\right.$ mbar working conditions, $10^{-10} \mathrm{mbar}$ base pressure). In order to have a homogeneous deposition and to reach the expected stoichiometry, the samples are rotated continuously around their surface normal during the deposition while kept at a temperature of $\sim 900 \mathrm{~K}$. The oxide deposition rate was about $0.12 \mathrm{~nm} / \mathrm{min}$. To investigate the quality and the structure of the grown epitaxial films, in situ reflection high-energy electron diffraction (RHEED) patterns were acquired during the film growth. The thickness of the $\mathrm{BaTiO}_{3}$ layers investigated in the present work lies in-between 15 and $30 \mathrm{~nm}$. In this range no influence of thickness on any measurements could be observed within the techniques used in this paper which is noticeably consistent with the previous report by Kim et al. [22] who reported a critical thickness for ferroelectricity of $\mathrm{BaTiO}_{3}$ of $4 \mathrm{~nm}$ and very little changes of the remnant polarization with thickness above $15 \mathrm{~nm}$.

In situ XPS spectra of the Ba 3d, Ti $2 p$ and 01 s core levels, using Al- $\mathrm{K}_{\alpha}$ radiation, were systematically recorded just after deposition to check the consistency of the $\mathrm{BaTiO}_{3}$ layer stoichiometry with previous reports [21]. XPS spectra were also recorded after the different polarization treatments to check eventual compositional changes in the layers.

We used Atomic Force Microscopy (AFM) and Piezoresponse Force Microscopy (PFM) to investigate the surface morphology and ferroelectric properties of our samples respectively. The images were realized with a Brüker ${ }^{\mathrm{TM}}$ scanning probe microscope and a Nanoscope $\mathrm{V}$ controller.

The samples were electrically poled using two major strategies as depicted schematically in Fig. 1. (i) Local poling (microscopic scale) was achieved using PFM, which was used in writing mode to create well defined ferroelectric domains in our samples, by poling with various positive or negative potentials applied on the tip (in the $[0 \mathrm{~V}$, $10 \mathrm{~V}]$ range), Fig. 1a and b. Since, the back of the sample was always connected to the ground, the sample surface was thus exposed to potentials in the $[-10 \mathrm{~V},+10 \mathrm{~V}]$ range. This poling procedure will be hereafter referred to as PFM-poling. The tip velocity was $0.4 \mu \mathrm{m} / \mathrm{s}$ and the writing resolution was $20 \mathrm{~nm}$. (ii) Macroscopic poling was realized using a non-aqueous $\mathrm{LiClO}_{4}$ electrolyte $\left(0.1 \mathrm{M} \mathrm{LiClO}_{4}\right.$ in propylene carbonate), with a large electrochemical window (i.e. a large voltage range in between which the substance is neither oxidized nor reduced), in an electrochemical cell with 2 electrodes. The sample was mounted as the working electrode and a Pt wire as the counter electrode. The geometry of the experimental setup is shown on Fig. 1c and $d$ and a scheme of the experiment is given on Fig. 1e. This poling procedure will be hereafter referred to as EC-poling. During the EC-poling procedure, the sample was kept at $0 \mathrm{~V}$. Potentials of $+8 \mathrm{~V}$ and $-8 \mathrm{~V}$ were applied several times alternatively (while monitoring the current) on the platinum counter electrode (during $10 \mathrm{~s}$ each) in order to reinforce the ferroelectric state as proposed by Roelofs et al. [23]. To obtain a remnant electric polarization pointing towards the substrate (by convention called downward polarization), the last applied potential was $+8 \mathrm{~V}$. Indeed, in a previous work [5], we demonstrated that $+8 \mathrm{~V} \mathrm{EC}$-poling leads to downward polarization of the $\mathrm{BaTiO}_{3}$ layer. Lastly the electric current monitoring is of crucial importance during EC-poling since overcurrent appeared to be strongly detrimental to the samples as we will demonstrate later in this paper.

The PFM-poled written ferroelectric domains were observed by PFM and Low Energy Electron Microscopy (LEEM). Beyond the recognized full field morphology characterization capabilities of LEEM [24,25], it has been previously reported that surface charge changes, expected when the ferroelectric polarization orientation changes, provide a visible LEEM contrast [26]. This phenomenon was used to observe ferroelectric contrasts between polarized and unpolarized regions of the sample regions. The samples were optically patterned by laser lithography and e-beam evaporation to include metallic Au landmarks on the sample surface that allow finding the PFM polarized regions easily. The $200 \mathrm{~nm}$ thick Au landmarks were evaporated on top of a $15 \mathrm{~nm}$ thick Ti seed layer. For a broader view, one may also use Kelvin probe force microscopy $[27,28]$ or scanning surface potential microscopy [29] to map the surface charge changes due to different ferroelectric domains polarization orientations, and DARTSSPFM for P-E loops measurements [30].

The high chemical stability in air of $\mathrm{BaTiO}_{3}$, as well as of the $\mathrm{Nb}: \mathrm{SrTiO}_{3}$ substrates, allows for air annealing at high temperature without composition changes. Since annealing above the ferroelectric orderdisorder temperature is easily possible in air, it appears as a method of choice to "reset" the ferroelectric domains orientation after PFM- or EC-poling. This was realized in a high temperature furnace above the Curie temperature of $\mathrm{BaTiO}_{3}$ with typical rates of $14{ }^{\circ} \mathrm{C} / \mathrm{min}$ up to $280^{\circ} \mathrm{C}$.

\section{Results and discussion}

\subsection{Growth}

In situ RHEED patterns were observed and acquired during film growth to monitor the quality and the structure of the grown epitaxial samples. Upon growth, the RHEED patterns switched from features typical of the perovskite $\mathrm{Nb}: \mathrm{SrTiO}_{3}$ substrate to the ones characteristic of the $\mathrm{BaTiO}_{3}$ thin epitaxial film (Fig. 2), similarly to observations reported using undoped $\mathrm{SrTiO}_{3}$ (001) substrates [21]. By convention we indexed the reciprocal space using the reciprocal space unit vectors $(10)^{*}$ and $(01)^{*}$ to describe the primitive reciprocal surface lattice as depicted in Fig. 2e. The RHEED reciprocal space streaks remain mostly 2D rod-like with some hints of island formation for high thicknesses (intensity modulations along the streaks at high thickness). The surface lattice parameter can easily be extracted from the streaks position while the error bar is derived from the parameter dispersion obtained using several azimuths. The surface lattice parameter evolution (Fig. 2f) reveals an almost complete structural relaxation for thicknesses above $10 \mathrm{~nm}$. From our RHEED observations we can conclude that the layers are mainly epitaxial, single crystalline and flat.
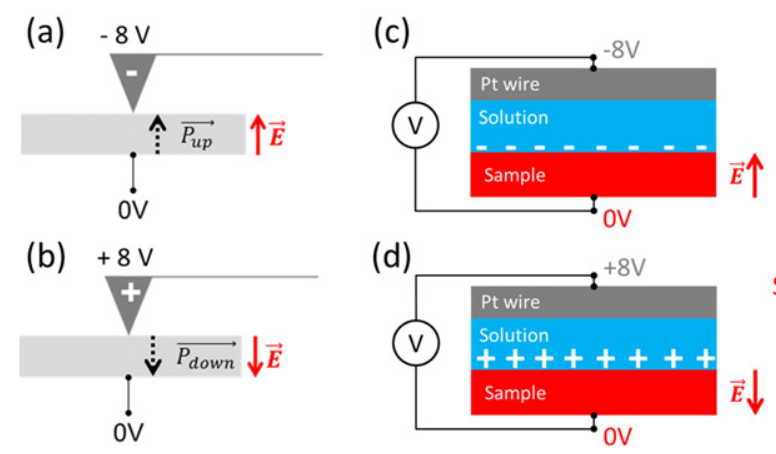

\section{(e)}

(d)
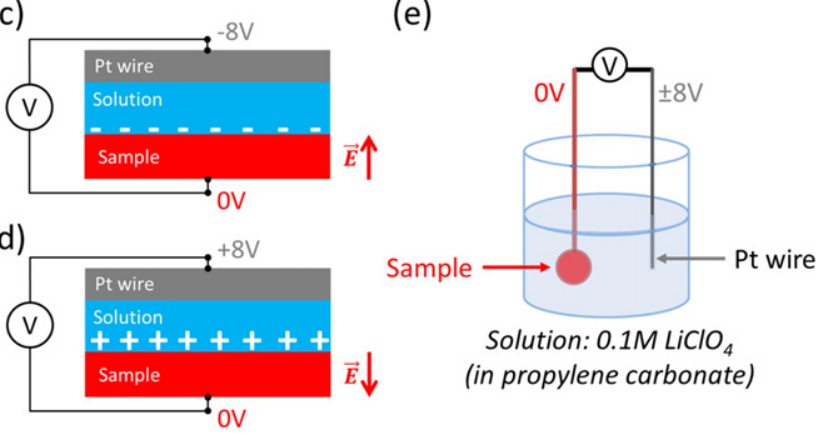

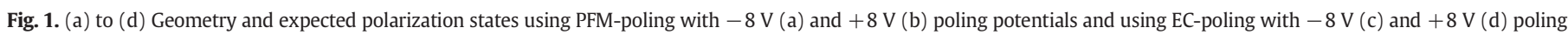
potentials. (e) Scheme of the EC-poling experiment. 

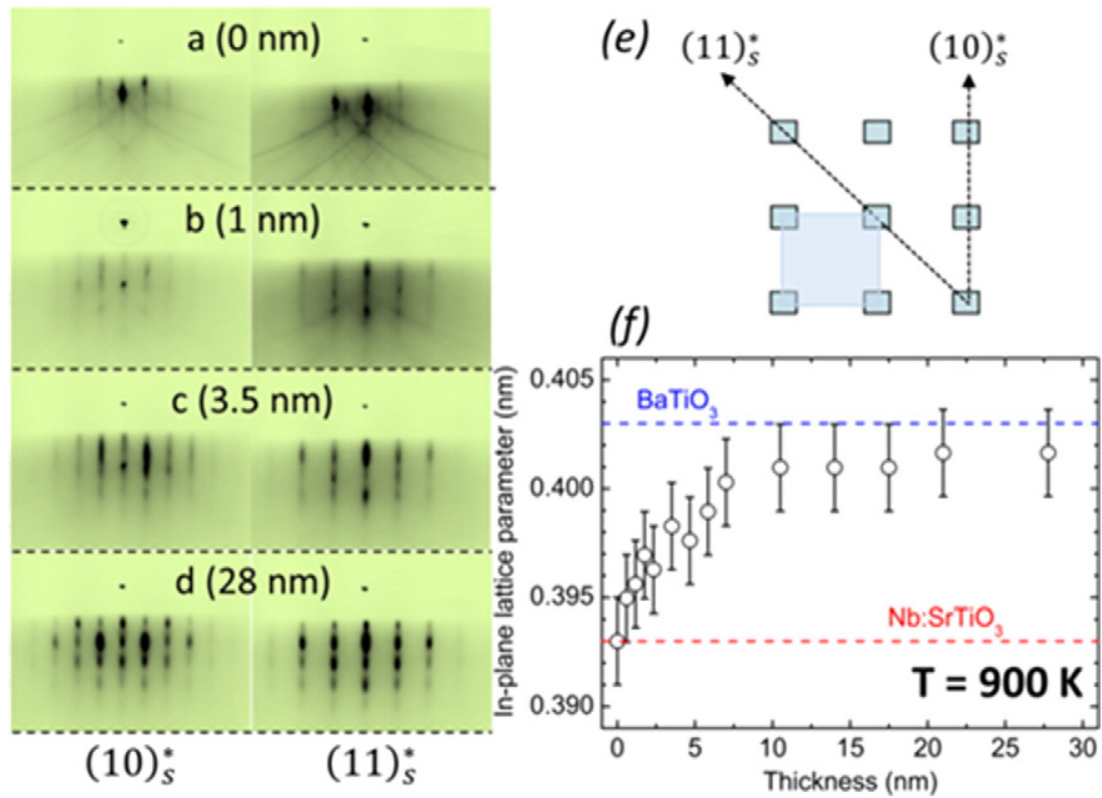

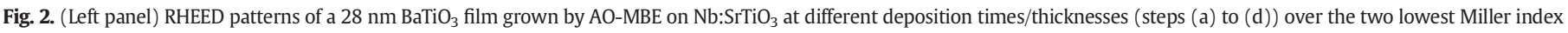

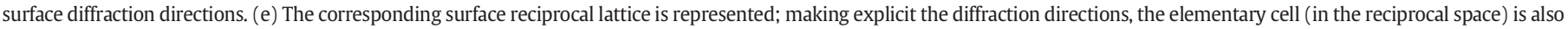

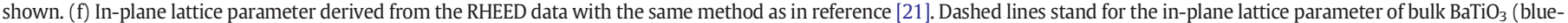
top) and $\mathrm{Nb}: \mathrm{SrTiO}_{3}$ (red-bottom) at the deposition temperature of $900 \mathrm{~K}$ (taking into account the thermal expansion).

The chemical composition of the as-grown layers was investigated by in situ XPS. A wide scan recorded over the entire accessible binding energy region (Fig. 3 ) shows the presence of the expected species ( $\mathrm{Ti}$, $\mathrm{O}$ and $\mathrm{Ba}$ ) and no additional eventual surface contamination. As previously reported in reference [21] a Ba rich surface oxide is necessary to promote a good epitaxial growth leading to $\mathrm{Ba}$ /Ti ratio above unity (typically $~ 1.3$ ). The binding energy of the Ba $3 d^{5 / 2}$ line was found at $780.4 \mathrm{eV}$ from detailed measurements of the Ba $3 d$ XPS lines as reported in Fig. 4.

\subsection{Microscopic polarization}

The ferroelectric nature of the layers was investigated by PFM. Scanning the tip-surface potential in the $[-10 \mathrm{~V},+10 \mathrm{~V}]$ range evidences two distinct polarization states. In order to determine the ferroelectric domain repartition we locally polarized the sample by applying $+8 \mathrm{~V}$

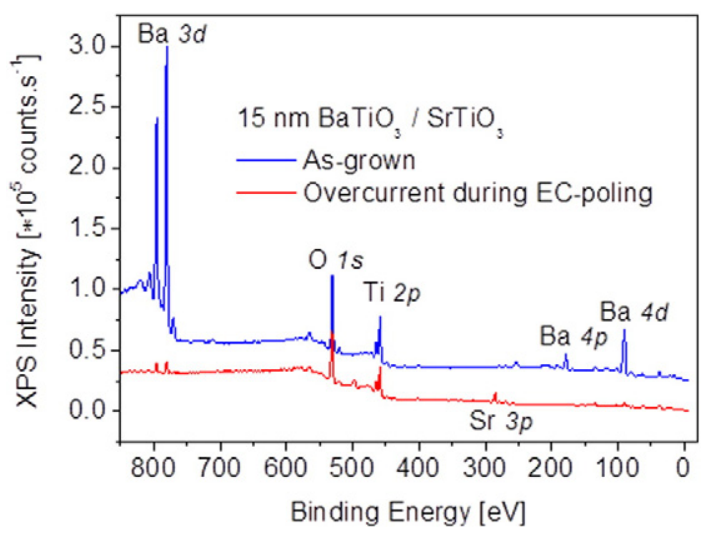

Fig. 3. Wide XPS scan of a $15 \mathrm{~nm}$ thick $\mathrm{BaTiO}_{3} / \mathrm{Nb}: \mathrm{SrTiO}_{3}$ (001) sample elaborated by AOMBE. ((blue, top curve) As grown sample and (red, bottom curve) for the same sample having experienced overcurrent during the macroscopic EC-poling procedure. The $01 s$ line (located at ca. $530.1 \mathrm{eV}$ ) of the as-grown sample was used as a reference position and the curves were shifted vertically for the sake of clarity. and $-8 \mathrm{~V}$ on the tip (substrate at ground) accordingly to the pattern shown in Fig. 5a. After the PFM-poling the pattern was read again by PFM using a sinusoidal potential applied on the sample (tip at ground) with a drive amplitude of $4000 \mathrm{mV}$. The resulting PFM phase image is shown in Fig. 5b. The integrated phase profile through the image of the written pattern (Fig. 5c) shows 3 different phase levels $\left(58^{\circ},-60^{\circ}\right.$ and $54^{\circ}$ ) corresponding to the different remnant states: (i) $+8 \mathrm{~V}$ PFM-poled, (ii) $-8 \mathrm{~V}$ PFM-poled and (iii) outside the writing region (i.e. $0 \mathrm{~V}$ or 'as-grown'), respectively. One may note that in this case the PFM phase of the as-grown region $\left(54^{\circ}\right)$ is close to the one after $+8 \mathrm{~V}$ polarization $\left(58^{\circ}\right)$. This result shows that the as-grown state behaves more like the $+8 \mathrm{~V}$ polarization states rather than like the $-8 \mathrm{~V}$ ones and that the ferroelectric domain mix between up and down orientations is different in each region. Moreover we observe that the PFM phase difference between the $+8 \mathrm{~V}$ and $-8 \mathrm{~V}$ remnant states is about $118^{\circ}$, which is smaller than the expected $180^{\circ}$ value. This phenomenon has been examined in more details in previous reports and can have

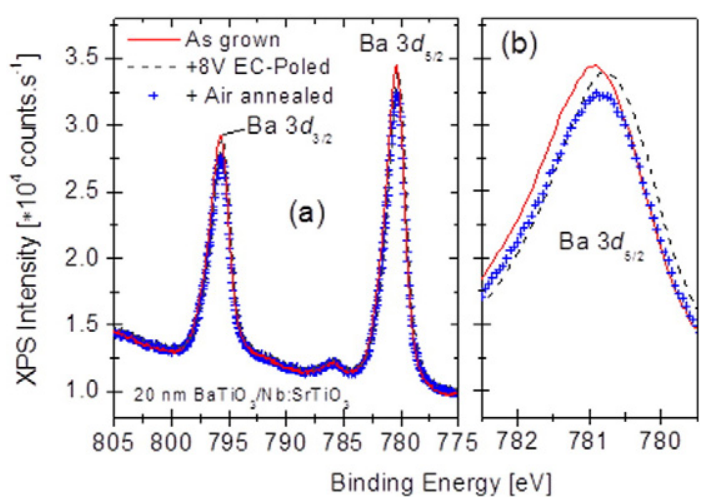

Fig. 4. $\mathrm{Ba} 3 d \mathrm{XPS}$ photoemission lines (a) recorded for a $20 \mathrm{~nm} \mathrm{BaTiO} / \mathrm{Nb}_{3} \mathrm{SrTiO}_{3}(001)$ layer grown by AO-MBE and (b) enlarged region of the main Ba $3 d_{5 / 2}$ line: $(-$, red solid line) as-grown layer, (-, black dashed line) after $+8 \mathrm{~V} \mathrm{EC}$-poling in a $\mathrm{LiClO}_{4}$ bath and ( + , blue cross-plus) after $+8 \mathrm{~V} \mathrm{EC}$-poling and a subsequent annealing in air at $280{ }^{\circ} \mathrm{C}$. 

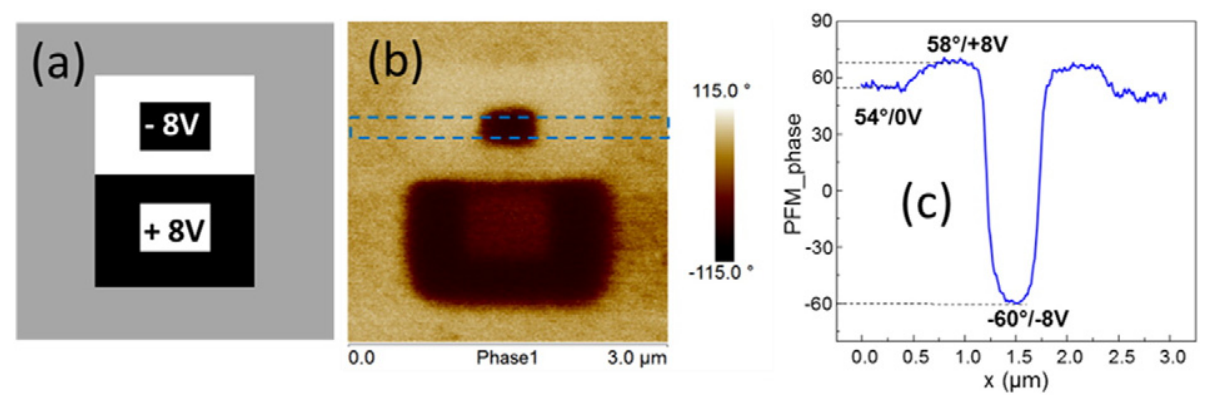

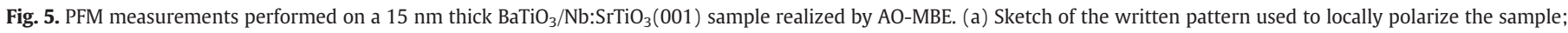

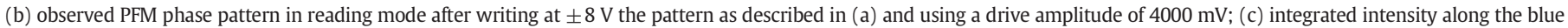
dashed box depicted in (b).

different origins: (i) the domains are not fully polarized (i.e. a mix of upward and downward domains); (ii) the PFM signal is disturbed by a background signal [31] inherent to the PFM setup; (iii) an asymmetry in polarization orientation may arise from an internal built-in electric field at the bottom interface which may result in incomplete $180^{\circ}$ switching [32]; and/or the drive amplitude was large enough to induce a charge build-up. Although the later effect is unlikely since successive scans did not evidenced any evolution of the patterns. However, one may note that an improved contrast using lower drive amplitude may be obtained thanks to the DARTSSPFM method [30], unfortunately this mode was not available in our setup. From region to region or sample to sample (within a set of about 10 samples) the observed domain mix, in the as-grown region, spans a wide range from nearly equiprobable to almost single domain. We can thus conclude that for the asgrown samples, the ferroelectric domains are arranged in a random fashion, i.e. a mix of upward and downward states.

PFM written patterns were subsequently investigated using full field LEEM imaging. Contrarily to PFM that can be used for writing and reading patterns, the LEEM technique allows a rapid investigation of large areas of the sample without eventual unwanted modifications as could be induced by a scanning tip, like during PFM measurements. Prior to LEEM imaging the sample was outgassed for several hours at $80{ }^{\circ} \mathrm{C}$. Working close to the Mirror Electron Microscopy (MEM) to LEEM transition (starting voltage close to $1 \mathrm{~V}$ ) allows the best imaging conditions and contrast. Thanks to the Au landmarks, the written pattern can easily be located as it can be seen in Fig. $6 \mathrm{~b}$ ( $50 \mu \mathrm{m}$ field of view (FOV). A closer inspection with a FOV of $10 \mu \mathrm{m}$ (Fig. 6c) confirms the presence of 3 different contrasts and thus the mixed initial polarization states of the as-grown samples. The LEEM observations are fully consistent with the previous PFM analysis.

\subsection{Macroscopic polarization}

After a PFM-poling (using the pattern shown on Fig. 6a), the sample was macroscopically EC-poled by keeping the current below $1 \mathrm{~mA}$ and observed again by LEEM. As it can be seen in Fig. 6d the pattern completely vanished upon this $+8 \mathrm{~V}$ EC-poling procedure, indicating that the macroscopic polarization overcomes the PFM writing. To confirm that the ferroelectric properties of the sample were unaltered by the EC-poling we performed again a PFM-poling using the initial PFM pattern in identical conditions and observed it subsequently by LEEM. As it can be seen in Fig. 6e the pattern is clearly visible defining again 3 contrasts states $(+8 \mathrm{~V}$ remnant state, $-8 \mathrm{~V}$ remnant state and ECpoled remnant states). In addition one may note that the surface morphology does not evolve upon EC-poling, remaining smooth and flat. Moreover, as revealed by XPS the composition remained unchanged after EC-poling since the peak intensities only marginally varied (Fig. 4). The peak position of the Ba $3 d^{5 / 2}$ line (Fig. 4b) is shifted to $780.2 \mathrm{eV}$. This shift is likely linked to a global surface charge effect although it is only slightly above the experimental error bar. Therefore we can conclude that EC-poling a $\mathrm{BaTiO}_{3}$ epitaxial layer in $\mathrm{LiClO}_{4}$ while keeping the current below $1 \mathrm{~mA}$ during the poling procedure changes neither the surface composition nor the morphology of the sample.

\subsection{Overcurrent}

By studying EC-poling with different successive increasing currents flowing through the sample, we found out that a current flow above $1 \mathrm{~mA}$ leads to detrimental results. We investigated in more details the effect of not limiting the current flowing through the sample during the EC-poling procedure. AFM images of a sample having experienced EC-poling without limiting the current (overcurrent) is reported in Fig. 7. During the EC-poling procedure, the sample was mounted in a special sample holder and fixed with a sealing joint in order to avoid the electrolyte to be in contact to the back of the substrate. As a matter of fact, the macroscopic polarization of the samples required some particular attention. Since $\mathrm{BaTiO}_{3}$ is ferroelectric and in consequence necessarily also piezoelectric, a mechanical strain may compete or even overcome the effect of an external electric field when one desires to tune the internal ferroelectric polarization. It is thus mandatory to get rid of any mechanical strain while performing an external poling procedure. In the case of our EC-poling procedure this was achieved by etching out a ring down to the substrate on the sample that corresponds to
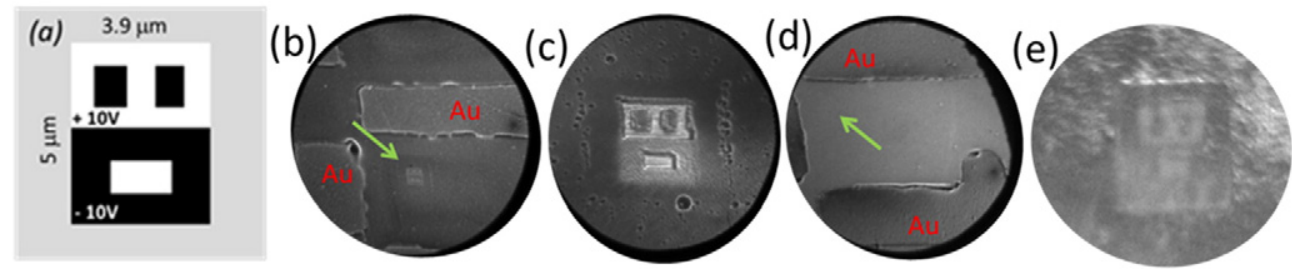

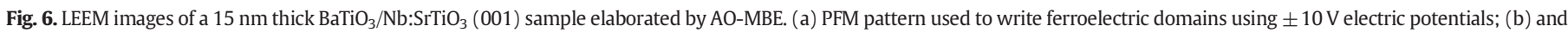

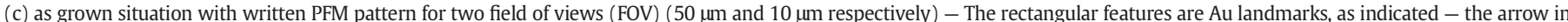

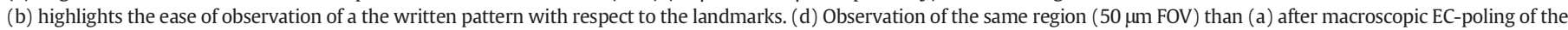

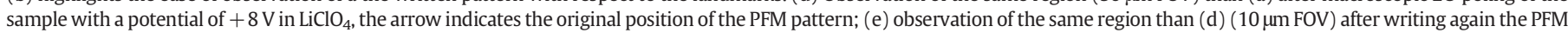
pattern depicted in (a) after EC-poling ( $10 \mu \mathrm{m}$ FOV). All images were taken around the MEM-LEEM transition potential with starting voltages ranging from 0.8 to $1.2 \mathrm{~V}$. 

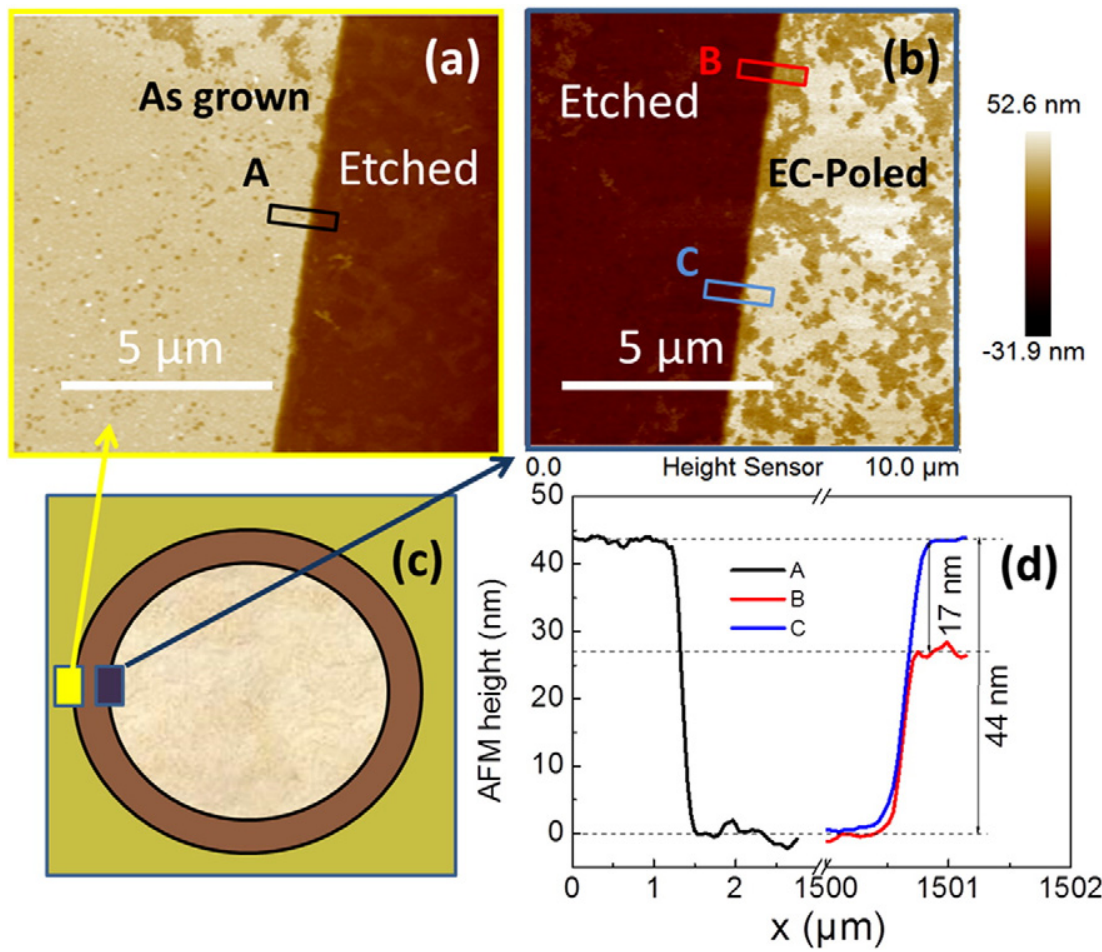

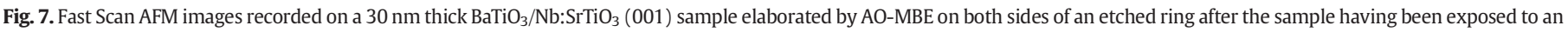

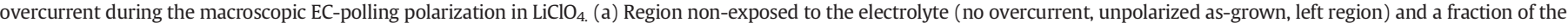

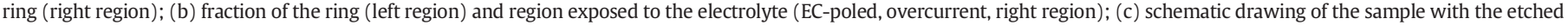
ring and (d) integrated step heights as measured on zones labeled A, B and C in (a) and (b).

the position of the electrochemical cell sealing joint (Fig. 7). The etched ring for sealing purpose separates the sample in two regions: outside the ring the $30 \mathrm{~nm}$ thick $\mathrm{BaTiO}_{3}$ layer was not exposed to the electrolyte (Fig. 7a) while the inside region which was EC-poled in $\mathrm{LiClO}_{4}$ was the siege of eventual overcurrent situations (Fig. 7b). This ring patterning allows comparing, on the same $\mathrm{BaTiO}_{3}$ sample, surfaces which underwent overcurrent upon EC-poling (Fig. 7b, inside) or not (Fig. 7a, outside). Integrated topography profiles at locations corresponding to different contrasts (Fig. 7d) show that the difference of height between the $B$ and $C$ regions is around $17 \mathrm{~nm}$. Since the nominal thickness of this $\mathrm{BaTiO}_{3}$ film was $30 \mathrm{~nm}$, we can conclude that the darker regions correspond to sample zones where the $\mathrm{BaTiO}_{3}$ film partially disappeared. The film morphology and thickness seem unperturbed in the lighter (unexposed) regions. The corresponding global XPS scan (Fig. 3) recorded on this sample confirms the massive loss of $\mathrm{BaTiO}_{3}$ after the overcurrent exposure of the surface, which is most likely due to the dissolution in the electrolyte.

\subsection{Annealing}

Macroscopic single polarization states as likely produced by ECpoling may be interesting for specific applications. For example we could show a 2.5 times increased photocurrent density during solar water splitting using a polarized $\mathrm{BaTiO}_{3}$ layer with respect to a nonpolarized configuration [5]. However, for many aspects, it is very desirable to be able to reset the sample domain configuration. To ensure the complete control of the ferroelectric polarization and the repeatability of our different poling procedures, we performed an air annealing of an EC-poled samples at $280{ }^{\circ} \mathrm{C}$ (above the Curie temperature of $\mathrm{BaTiO}_{3}$ ). After annealing we could write again patterns using PFM indicating that after crossing the Curie temperature the sample came back to a more mixed polarization state (effective ferroelectric polarization "reset"). XPS showed unchanged composition and the Ba $3 d^{5 / 2}$ lines where found at a position intermediate between the as-grown situation and the EC-poled ones (Fig. 4b). The ability to reset the polarization state was investigated in more details by PFM starting from a virgin sample. The ferroelectric domain configuration of a $15 \mathrm{~nm}$ thick $\mathrm{BaTiO}_{3} / \mathrm{Nb}: \mathrm{SrTiO}_{3}$ layer was investigated before and after annealing in air at $280^{\circ} \mathrm{C}$ for $20 \mathrm{~min}$ (Fig. 8). The sample possessed Au landmarks, realized by lithography, to allow a precise positioning of the tip on a given region that was observed prior (Fig. 8a) and after writing (Fig. 8b). The very same region was imaged again after a sample annealing realized at $280{ }^{\circ} \mathrm{C}$ for $20 \mathrm{~min}$. As can be seen on Fig. $8 \mathrm{c}$ the pattern was "erased" after the annealing procedure. Finally, we checked that the sample showed similar ferroelectric behavior after annealing by writing a pattern on another region of the same sample, as observed in Fig. 8d. From these observations we can conclude that after an annealing cycle the ferroelectric configuration of the layer is "reset" to a new mixed polarization state configuration.

\section{Conclusion}

In conclusion, we have demonstrated efficient methods to manipulate the polarization state of a prototypical $\mathrm{BaTiO}_{3}$ ferroelectric layer and also to retrieve a new mixed polarization state after macroscopic polarization. We showed that our layers can be locally polarized using PFM within the $[-10 \mathrm{~V} ;+10 \mathrm{~V}]$ potential range. We have also shown that the $\mathrm{BaTiO}_{3}$ layer can be downward polarized macroscopically in a solution of $\mathrm{LiClO}_{4}$ in propylene carbonate chloride, provided that the current is maintained below $1 \mathrm{~mA}$. Upon overcurrent the $\mathrm{BaTiO}_{3}$ layer is likely to dissolve. Moreover, we found that an air annealing at a temperature above the Curie temperature performs a sort of "reset" of the ferroelectric polarization. Our study presented a set of tools in order to master the polarization states of ferroelectric layers, which is of top interest in the framework of the use of ferroelectric materials in new devices having tremendous potential in many technologically important fields like multiferroics for spintronics or photoelectrochemical water splitting devices in particular. 

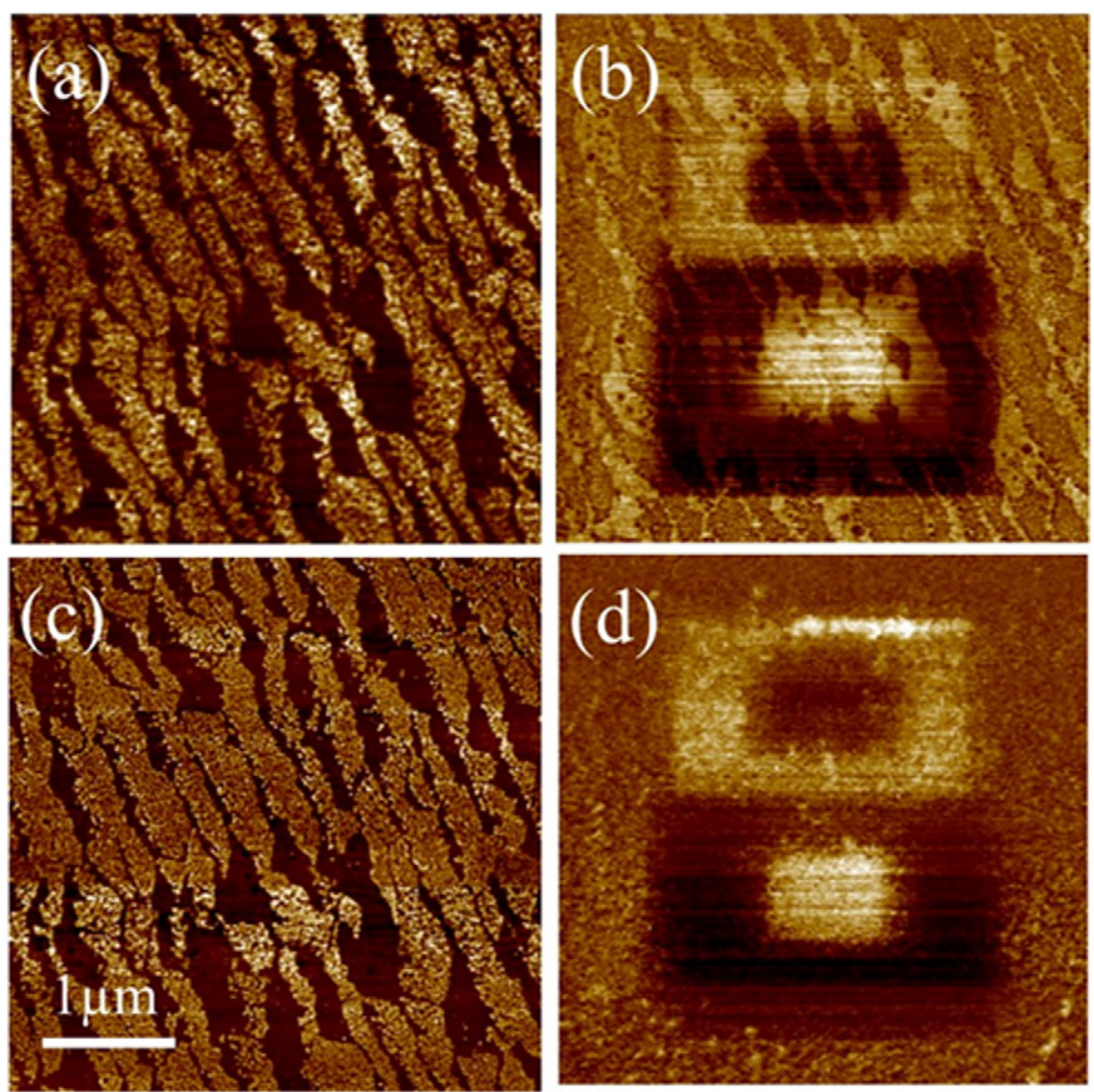

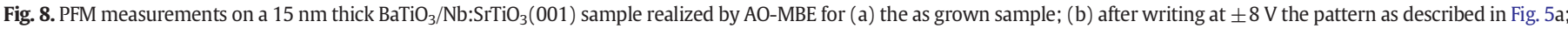
(c) same region than (b) observed after a sample annealing at $280{ }^{\circ} \mathrm{C}$ for $20 \mathrm{~min}$ and (d) after writing again the pattern on the sample on a virgin region.

\section{Conflict of interest}

The authors declare no conflict of interest.

\section{Acknowledgements}

This work was funded by the CEA (DSM-Energie program under the PAPI grant) and supported in part by Triangle de la Physique and Ile-deFrance (C'Nano and ISC-PIF) under the IMAMFP grants.

\section{References}

[1] Y. Tokunaga, N. Furukawa, H. Sakai, Y. Taguchi, T.-H. Arima, Y. Tokura, Composite domain walls in a multiferroic perovskite ferrite, Nat. Mater. 8 (2009) 558.

[2] Evgeny Y. Tsymbal, Elbio R.A. Dagotto, Chang-Beom Eom, Ramamoorthy Ramesh, Multifunctional Oxide Heterostructures, OUP Oxford, 2012 ISBN: 9780199584123.

[3] N. Jedrecy, H.J. von Bardeleben, V. Badjeck, D. Demaille, D. Stanescu, H. Magnan, A Barbier, Strong magnetoelectric coupling in multiferroic $\mathrm{Co} / \mathrm{BaTiO} 3$ thin films, Phys. Rev. B 88 (2013) 121409(R).

[4] A. Barbier, T. Aghavnian, V. Badjeck, C. Mocuta, D. Stanescu, H. Magnan, C.L. Rountree, R. Belkhou, P. Ohresser, N. Jedrecy, Antiferromagnetic long-range spin ordering in Fe- and NiFe2-doped BaTiO3 multiferroic layers, Phys. Rev. B 91 (2015) 035417.

[5] M. Rioult, S. Datta, D. Stanescu, S. Stanescu, R. Belkhou, F. Maccherozzi, H. Magnan, A. Barbier, Tailoring the photocurrent in BaTiO3/Nb:SrTiO3 photoanodes by controlled ferroelectric polarization, Appl. Phys. Lett. 107 (2015) 103901.

[6] W. Ji, K. Yao, Y.F. Lim, Y.C. Liang, A. Suwardi, Epitaxial ferroelectric BiFeO3 thin films for unassisted photocatalytic water splitting. Appl. Phys. Lett. 103 (2013) 062901.

[7] K. Meng, P.K. Surolia, K.R. Thampi, BaTiO3 photoelectrodes for CdS quantum dot sensitized solar cells, J. Mater. Chem. A 2 (2014) 10231.

[8] S. Park, C.W. Lee, M.-G. Kang, S. Kim, H.J. Kim, J.E. Kwon, S.Y. Park, C.-Y. Kang, K.S. Hong, K.T. Nam, A ferroelectric photocatalyst for enhancing hydrogen evolution: polarized particulate suspension, Phys. Chem. Chem. Phys. 16 (2014) 10408.

[9] G. Dong, H. Fan, H. Tian, J. Fang, Q. Li, Gas-sensing and electrical properties of perovskite structure p-type barium-substituted bismuth ferrite, RSC Adv. 5 (2015) 29618.
[10] A. Navrotsky, Thermochemistry of perovskite-related oxides with high oxidation states: superconductors, sensors, fuel cell materials, Pure Appl. Chem. 66 (1994) 1759 .

[11] T. Tasaki, S. Takase, Y. Shimizu, Fabrication of Sm-based perovskite-type oxide thinfilms and gas sensing properties to acetylene, J. Sens. Technol. 2 (2012) 75.

[12] K.Y. Yun, D. Ricinschi, T. Kanashima, M. Noda, M. Okuyama, Giant ferroelectric polarization beyond $150 \mu \mathrm{C} / \mathrm{cm}^{2}$ in BiFeO3 thin film, Jap. J. Appl. Phys. 43 (2004) L647.

13] S.Y. Yang, F. Zavaliche, L. Mohaddes-Ardabili, V. Vaithyanathan, D.G. Schlom, Y.J. Lee, Y.H. Chu, M.P. Cruz, Q. Zhan, T. Zhao, R. Ramesh, Metalorganic chemical vapor deposition of lead-free ferroelectric BiFeO3 films for memory applications, Appl. Phys. Lett. 87 (2005) 102903.

[14] K. Iijima, T. Terashima, K. Yamamoto, K. Hirata, Y. Bando, Preparation of ferroelectric BaTiO3 thin films by activated reactive evaporation, Appl. Phys. Lett. 56 (1990) 527.

[15] K.S. Young, K.S. Kim, Y.D. Park, J.-G. Yoon, Critical thickness of ultrathin ferroelectric BaTiO3 films, Appl. Phys. Lett. 86 (2005) 102907.

[16] J. Fukushima, K. Kodaira, T. Matsushita, Preparation of ferroelectric PZT films by thermal decomposition of organometallic compounds, J. Mater. Sci. 19 (1984) 595.

[17] R. Moazzami, C. Hu, W.H. Shepherd, Electrical characteristics of ferroelectric PZT thin films for DRAM applications, IEEE Trans. Electron Devices 39 (1992) 9.

[18] M. Singh, Y. Yang, C.G. Takoudis, A. Tatarenko, G. Srinivasan, P. Kharel, G. Lawes, Metallorganic chemical-vapor-deposited $\mathrm{BiFeO3}$ films for tunable high-frequency devices, Electrochem. Solid-State Lett. 12 (2009) H161.

[19] D. Cao, Z. Wang, L. Nasori, Y. Wen, Mi, Y. Lei, Switchable charge-transfer in the photoelectrochemical energy-conversion process of ferroelectric $\mathrm{BiFeO} 3$ photoelectrodes, Angew. Chem. 126 (2014) 11027.

[20] K.J. Choi, M. Biegalski, Y.L. Li, A. Sharan, J. Schubert, R. Uecker, P. Reiche, Y.B. Chen, X.Q. Pan, V. Gopalan, L.-Q. Chen, D.G. Schlom, C.B. Eom, Enhancement of ferroelectricity in strained BaTiO3 thin films, Science 306 (2014) 1005.

[21] A. Barbier, C. Mocuta, D. Stanescu, P. Jegou, N. Jedrecy, H. Magnan, Surface composition of BaTiO3/SrTiO3(001) films grown by atomic oxygen plasma assisted molecular beam epitaxy, J. Appl. Phys. 112 (2012) 114116.

[22] Y.S. Kim, D.H. Kim, J.D. Kim, Y.J. Chang, T.W. Noh, J.H. Kong, K. Char, Y.D. Park, S.D. Bu, J.-G. Yoon, J.-S. Chung, Critical thickness of ultrathin ferroelectric BaTiO3 films, Appl Phys. Lett. 86 (2005) 102907.

[23] Andreas Karl Roelofs, Martin Gerard Forrester and Joachim Walter, US patent US 2007/0041233 A1 (2007)

[24] E. Bauer, Low energy electron microscopy, Rep. Prog. Phys. 57 (2014) 895-938 (R).

[25] E. Bauer, Low-Energy Electron Microscopy, in: G. van Tandeloo, Dirk Van Dyck, S.J. Pennycook (Eds.), Handbook of Nanoscopy, Wiley 2012, pp. 673-696 (R). 
[26] J.E. Rault, W. Ren, S. Prosandeev, S. Lisenkov, D. Sando, S. Fusil, M. Bibes, A. Barthelemy, L. Bellaiche, N. Barrett, Thickness-dependent polarization of strained BiFeO3 films with constant tetragonality, Phys. Rev. Lett. 109 (2012) 267601.

[27] Y. Kim, S. Bühlmann, J. Kim, M. Park, K. No, Local surface potential distribution in oriented ferroelectric thin films, Appl. Phys. Lett. 91 (2007) 052906.

[28] L. Wang, K.-J. Jin, J.-X. Gu, C. Ma, X. He, J. Zhang, C. Wang, Y. Feng, Q. wan, J.-A. Shi, M. He, H.-B. Lu, G.-Z. Yang, A new non-destructive readout by using photo-recovered surface potential contrast, Sci. Rep. 4 (2014) 6980.
[29] X.Q. Chen, H. Yamada, T. Horiuchi, K. Matsushige, S. Watanabe, M. Kawai, P.S. Weiss, Surface potential of ferroelectric thin films investigated by scanning probe microscopy, J. Vac. Sci. Technol. B 17 (1999) 1930.

[30] B.J. Rodriguez, C. Callahan, S.V. Kalinin, R. Proksch, Dual-frequency resonancetracking atomic force microscopy, Nanotechnology 18 (2007) 475504.

[31] T. Jungk, A. Hoffmann, E. Soergel, Quantitative analysis of ferroelectric domain imaging with piezoresponse force microscopy, Appl. Phys. Lett. 89 (2006) 163507.

[32] A. Gruverman, A. Kholkin, A. Kingon, H. Tokumoto, Asymmetric nanoscale switching in ferroelectric thin films by scanning force microscopy, Appl. Phys. Lett. 78 (2001) 2751. 